\title{
Modelos conceptuales que explican la discapacidad: de la teoría a la comprensión del funcionamiento
}

\section{MODELOS CONCEPTUALES Y EVOLUCIÓN HISTÓRICA DE LA DISCAPACIDAD}

La discapacidad es la quintaesencia del concepto postmoderno, porque es tan compleja, tan variable, tan contingente, tan situada. Está ubicada en la intersección de la biología y la sociedad y entre agencia y estructura. La discapacidad no puede ser reducida a una identificación singular, es una multiplicidad, una pluralidad.

(Shakespeare \& Watson) (1) 
La discapacidad es una realidad que ha sido comprendida y abordada de diferente manera a través de la historia. Cada vez con mayor fuerza se impulsa la necesidad de comprenderla desde una mirada más amplia en la que se reconoce que esta ya no es un problema del individuo, sino del entorno. Este proceso de evolución del concepto de discapacidad, obedece entre "otras razones, a la luz de las reflexiones teóricas y de los procesos históricos marcados por la presión de diferentes grupos sociales alrededor de la reivindicación de los derechos de este colectivo, lo que ha determinado escenarios de inclusión dentro del proyecto de sociedad" (2).

Así pues, reconocer la perspectiva conceptual de la discapacidad a través de la historia y de la forma en cómo esta se han transformado, obedece igualmente a los cambios en los paradigmas sociales e ideológicos que se tienen frente a las mismas personas, pues son ellas mismas las que han suscitado el cambio en el abordaje de la discapacidad. Por otro lado, dichas transformaciones también responden a la mirada que desde las ciencias se hace de la problemática de la discapacidad; por ejemplo, en las ciencias de la salud, sobre todo a partir de la segunda mitad del siglo XIX, se adopta una visión médica y terapéutica que enfatiza el déficit, el tratamiento, la curación y la rehabilitación de las distintas discapacidades. En las ciencias sociales, durante el siglo XX y lo que va del siglo XXI, la discapacidad cobra interés para la investigación desde los campos de la psicología, la educación y la sociología (3).

Sin embargo, tal como lo sugiere Gómez, "la investigación en discapacidad y ciencia social, no ha llegado a consenso sobre qué constituye la discapacidad". A partir de esta complejidad, la discapacidad en el plano conceptual y práctico ha tenido diferentes lecturas en razón a tiempos y espacios determinados (1).

Bajo esta perspectiva, surgen los llamados modelos de discapacidad, los 
cuales representan la forma en que es explicada y abordada esta realidad a través de la historia. Un modelo devela una construcción social frente a una situación en particular, que determina la forma en cómo la sociedad visibiliza la complejidad de las múltiples relaciones que se establecen en una situación determinada. "La noción de modelo se refiere a representaciones conceptuales y relacionales utilizadas para explicar y comprender la realidad de cierta manera, ya que perfilan aspectos relevantes a una problemática de estudio" (3). Ningún modelo en forma independiente, de acuerdo con Mitra, puede explicar totalmente la discapacidad, debido a que cada modelo depende del contexto en el que se proponga (1), y de la perspectiva teórica que lo explica.

Tal como lo propone Vargas, las distintas perspectivas teóricas y sus respectivos enfoques enmarcan distintos modelos sobre la discapacidad que discrepan en sus conceptualizaciones. La perspectiva teórica está determinada por la "aproximación epistemológica, entendida esta como la forma de acercarse a la realidad que se conoce y a la relación que en ese acercamiento se establece entre la persona cognoscente, el objeto del conocimiento y el conocimiento mismo". En este orden de ideas los sistemas teóricos, en tanto estructuras epistemológicas que dan cuenta de la discapacidad como un área del conocimiento, también son sistemas, que se relacionan con el fenómeno de la discapacidad, ya que ellos constituyen una forma de ver el mundo (4).

$\mathrm{Al}$ respecto, es diversa la literatura que existe alrededor de las últimas tres décadas, donde se reporta heterogeneidad de modelos conceptuales que permiten explicar la discapacidad pasando desde la perspectiva médica, salubrista, económica, política, administrativa hasta la perspectiva jurídica, lo cual deja ver el interés en dicho tema por parte de todas las sociedades, lo cual resulta en un ejercicio complejo y que genera muchas controversias 
que bien sea dicho, se requieren para la construcción y deconstrucción de conceptos que permitan explicar esta situación en particular que tiene implicaciones en todas las esferas de la vida de los pueblos (5).

Tal como lo propone Cortés, el ejercicio de conceptualización de la discapacidad se constituye un asunto medular en cuanto tiene implicaciones políticas y prácticas, como en la medición de prevalencia de discapacidad, formulación de políticas, construcción de sistemas de información, entre otros, que inciden en forma directa en la vida de las personas con discapacidades (2).

En tal sentido, los modelos conceptuales alrededor de una realidad, presuponen una comprensión de esa realidad en determinado momento histórico, que guía los procesos de abordaje e intervención de la misma. "Definir entonces los modelos conceptuales de la discapacidad permite vislumbrar las implicaciones políticas y prácticas de acuerdo con el abordaje adoptado, ya sea una ley o un programa de atención a personas en situación de discapacidad" (1).

De igual manera, un modelo conceptual en el área de la salud, por ejemplo, ofrece una "visión clara de los principios científicos y filosóficos que orientan su práctica profesional, para actuar en concordancia con ellos" (6). Dichos modelos reflejan la forma en que se establece la relación entre los diferentes profesionales y la persona con discapacidad, y de la forma en que esta se relaciona y desarrolla en la sociedad; lo anterior en respuesta a lo que la sociedad logra interpretar de la actuación del sujeto en el colectivo. Tal como lo propone la teoría cognitiva de la discapacidad, esta se construye a partir de las percepciones de los sujetos, que marcan la cimentación de los estereotipos sociales, lo cual avanza hacia la construcción social derivada de las representaciones que se van edificando en colectivo. 
A partir de todo lo anterior, subyacen diversas preguntas alrededor del tema: ¿Para qué comprender los modelos de discapacidad? ¿Qué horizonte en la intervención y/o abordaje de discapacidad ofrece la comprensión de los modelos de discapacidad? y ¿Cómo categorizarlos en un mar de modelos que se posicionan desde diversas categorías de análisis? Se pretende develar las respuestas a estos interrogantes en la medida en que se realice un análisis de los diferentes modelos para la comprensión de la discapacidad.

Los modelos teóricos alrededor de la discapacidad, se definen en función de diversas categorías y perspectivas teóricas de análisis, por eso son múltiples las clasificaciones que se encuentran en la literatura para abordar la discapacidad. A continuación, se muestran algunas taxonomías que se han definido en el intento por darle fundamento precisamente a la mirada y abordaje de la discapacidad:

- Peñas describe algunos modelos que han prevalecido a través de la historia, de caridad, médico, social, relacional y universalizante. En esta perspectiva no se define de manera particular una categoría específica de análisis.

- López González, citado por Velázquez, realiza una clasificación basada en la dialéctica entre individuo y sociedad, en la que encontraríamos: Concepciones de la discapacidad como condición individual; Perspectivas sociológicas: por un lado, versiones construccionistas sociales (funcionalistas, interaccionistas) y, por otro, versiones materialistas (creacionismo social); y Perspectivas (por las que aboga la autora para el futuro) basadas en el análisis de la discapacidad como experiencia personal (7).

- Por su parte Velázquez, a partir de la diferenciación que realiza entre paradigma y modelo, propone la siguiente taxonomía: (7) 
- El paradigma de la prescindencia. El modelo eugenésico y el modelo de la marginación.

- El paradigma de la rehabilitación. El modelo médico y el surgimiento del modelo "bio-psico-social" como reacción al modelo social.

- El paradigma de la autonomía personal. El modelo social y el modelo de la diversidad funcional.

- La OMS ha definido sus modelos desde la perspectiva de las relaciones que se tejen en el abordaje de la discapacidad, por lo que es claro identificar en sus referencias, el modelo médico, el modelo social y hoy el modelo biopsicosocial, sobre los cuales han emergido las clasificaciones que le son propias.

- Gómez (1), partiendo de la posición relacional de discapacidad, la cual se define como la condición resultante de la interacción entre individuo y ambiente, realiza un análisis del modelo médico y el modelo social, para presentar un análisis del modelo biopsicosocial de la OMS, como el modelo que media la conciliación entre estas dos posiciones; modelo que provee una perspectiva distinta de los estados de la salud bajo factores sociales y biológicos. Siguiendo su intencionalidad de ofrecer un modelo relacional de la discapacidad, también coloca a discusión el modelo presentado por el Instituto de Medicina de los Estados Unidos (IOM), el cual se soporta por la interacción entre una interacción entre el individuo y su ambiente, representado por condiciones psicológicas (plano interpersonal), hasta lo físico y lo social.

- Otros autores (8) presentan una taxonomía situada en el tratamiento social de la discapacidad, en el que es posible diferenciar, el modelo de prescindencia, el modelo rehabilitador, el modelo social 
y el modelo de la diversidad. Estos modelos son analizados por los autores como punto de partida para explicar un modelo emergente: El enfoque de capacidades y funcionamientos de Amartya Sen.

- La Universidad Autónoma de Manizales, en el marco de la fundamentación del proyecto educativo del Programa de Maestría en Discapacidad (9), alude una taxonomía de los modelos de discapacidad, articulando la concepción de cuerpo, con la perspectiva teórica y el modelo conceptual que le subyace:

- Cuerpo objetivo, una perspectiva de la discapacidad desde la teoría funcionalista estructuralista en el modelo médico-biológico.

- Cuerpo subjetivo, una perspectiva de la discapacidad desde la teoría interaccionista, en el modelo social.

- Cuerpo intersubjetivo, una perspectiva de la discapacidad desde la teoría socio-crítica en el modelo político-activista.

- Cuerpo sujeto y persona con discapacidad.

De acuerdo con Mitra, "la multitud de modelos pueden reflejar la naturaleza multifacética de la discapacidad" (p.236). El rango en las definiciones de discapacidad desarrollado hasta el momento ha partido desde enfoques opuestos centrados en la persona -en los cuales la deficiencia es la condición que lleva a la existencia de la discapacidad, como en el modelo médico- hasta visiones universalizantes en las cuales todos los seres humanos están en riesgo real de llegar a esta condición (1).

En respuesta a lo anterior, a continuación se hace una descripción y análisis de los modelos que han estado a la base de la comprensión y abordaje de la discapacidad, en la que se devela la teoría que le da fundamento, y la mirada del cuerpo en todas sus dimensiones que le subyace a la construcción social y paradigmática de la discapacidad. 


\section{EL MODELO BIOLÓGICO, UNA MIRADA INDIVIDUAL DE LA DISCAPACIDAD}

Se conoce también como modelo biomédico, modelo individual de la discapacidad o aproximación de la limitación funcional; es quizás el más reconocido y anclado en los paradigmas sociales. "Bajo este modelo la discapacidad se origina como una consecuencia de una patología activa y continúa incluso cuando la situación de salud se ha superado. En tal sentido define la discapacidad como la inhabilidad o limitación en el desempeño de roles socialmente definidos y tareas que se espera que pueda desempeñar en un determinado ambiente" (5).

En perspectiva social, este modelo explica que el único origen de la exclusión enfrentada por personas con discapacidad son sus propias limitaciones, ocasionadas por el mal funcionamiento del cuerpo. Así la problemática está situada en la persona, en tanto que reside en ella, como consecuencia de una enfermedad, trauma o lesión, y que se busca superarla a través de intervenciones terapéuticas individuales

El modelo médico de la discapacidad se centra en las limitaciones del cuerpo de la persona, por lo que "En este modelo se objetiva el cuerpo objetivo y la teoría funcionalista-estructuralista y se analiza la discapacidad como una de las consecuencias de la enfermedad, es decir, la enfermedad puede originar déficit en la función o la funcionalidad del sujeto que lo inhabilitan para el adecuado desempeño en la vida cotidiana" (9).

Este modelo define la discapacidad como la inhabilidad o limitación en el desempeño de roles socialmente definidos y tareas que se espera pueda desempeñar en un determinado ambiente (5). En tal sentido el modelo biológico sitúa su análisis únicamente en déficit corporal y sobre este promueve todas las medidas de intervención, que solo se limitan a resolver 
los problemas del cuerpo en la actuación en la vida cotidiana. "El objetivo de la actuación ha de ser la búsqueda de la adaptación del individuo con discapacidad a las demandas y exigencias de la sociedad. Para facilitar dicha adaptación, que en cualquier caso ha de ser personal e individual, se postula como objetivo fundamental de actuación, el establecimiento de medidas compensatorias que palien las deficiencias" (10).

El modelo médico es entendido en otras taxonomías como el modelo rehabilitador, en cuyo caso se alude en primer lugar a la discapacidad en términos de "enfermedad" o como "ausencia de salud", y en segundo lugar se considera que las personas con discapacidad pueden tener algo que aportar a la comunidad, pero solo en la medida en que sean rehabilitadas o normalizadas, y logren asimilarse a las demás personas (válidas y capaces) en la mayor medida posible. Con lo cual, entran en un "proceso de normalización" aun de poder obtener por parte de la sociedad un valor como personas y como ciudadanas y ciudadanos (8).

A partir de lo anterior, se suscita entonces la pregunta sobre las implicaciones de un modelo médico biológico en el abordaje de la discapacidad, el cual se puede resumir en lo siguiente:

- Solo la restauración de la función corporal tiene sentido, por tanto, solo la cura de la discapacidad puede traer dignidad y felicidad a la persona con discapacidad.

- En este escenario, basta con medidas rehabilitadoras situadas únicamente en la persona, por lo que la intervención de la sociedad queda relegada.

- Se trata en todo caso de prevenir la discapacidad como tragedia de las vidas de las personas, en tanto que esta situación es vista como una condición devastadora que limita las oportunidades de la persona en sociedad. 
- La adaptación debe entonces surgir de la persona hacia la sociedad, es el cuerpo el que debe adaptarse para desempeñarse en la vida en sociedad.

- El alcance de las políticas sanitarias centradas en el modelo médico, se agota en intervenciones paliativas, que se definen únicamente en ofrecer servicios de rehabilitación para el cuerpo, mas no para el sujeto en sociedad.

- Bajo este modelo, la persona con discapacidad asume dentro del proceso de rehabilitación un rol pasivo, en donde son los profesionales los que deben decidir cuál es el norte de la rehabilitación.

- El modelo se refleja claramente en la derivación de la política pública en nuestro país. La Ley 100 de 1993, la Ley 115 de 1993, son un ejemplo de la postura coincidente que adoptan frente al tema de discapacidad con los postulados del modelo médico, evidenciado en el énfasis que hacen en las limitaciones individuales, en el manejo de las deficiencias de la persona y entender la rehabilitación como la solución a la situación (11).

\section{EL MODELO SOCIAL, LA DISCAPACIDAD EMERGE EN SOCIEDAD}

El modelo social defiende que la discapacidad no es un atributo único de la persona situado en los déficits del cuerpo, en tanto que "no son las limitaciones individuales de las personas con discapacidad la causa del problema, sino las limitaciones de la sociedad para prestar los servicios apropiados y para garantizar que las necesidades de esas personas sean tenidas en cuenta dentro de la organización social. Esto no supone negar el aspecto individual de la discapacidad, sino enmarcarlo dentro del contexto social (8). Lo anterior representa el reconocimiento de la discapacidad como construcción social que depende directamente de la sociedad y que refleja el grado en que está preparada para intervenirla. 
Según la OMS la transformación de un modelo médico requirió pasar de una mirada individual y médica a una estructural y social donde se considera que la sociedad es la que discapacita a la persona, más que las limitaciones que esta presente para realizar actividades (12).

El desarrollo y comprensión de la discapacidad está ligada estrechamente por acontecimientos políticos, sociales, culturales y de orden económico que se dan en cada momento de la historia de la humanidad; desde esta mirada, desde los años 60 y 70 en países como Estados Unidos, Gran Bretaña, Países Escandinavos y España, se observaron movimientos sociales por comunidades de raza negra, minorías étnicas, mujeres y personas con discapacidad, las cuales presentan la exclusión a la que se ven expuestos y solicitan reconocimiento de sus derechos como seres que forman parte de la sociedad civil (13).

Este modelo ha evolucionado en términos de las relaciones que se establecen para la comprensión de la discapacidad. En este modelo, la discapacidad supera la mirada únicamente en el cuerpo, para pasar a una mirada del cuerpo en sociedad. Bajo esta perspectiva, "la discapacidad no es solo la consecuencia de los déficits existentes en la persona, sino resultante de un conjunto de condiciones, actividades y relaciones interpersonales, muchas de las cuales están motivadas por factores ambientales" (9).

El modelo social sitúa el análisis en dos aspectos fundamentales (13): por un lado en el cuerpo, ya no para identificar los aspectos anatómicos y su funcionamiento sino para revelar las habilidades y capacidades de la persona a partir de la limitación que presenta para buscar los medios para potencializarla; por el otro, analiza el entorno inmediato, dentro del cual sitúa la familia y el entorno social en los cuales se desenvuelve la persona con discapacidad. 
A partir de lo anterior, se suscita entonces la pregunta sobre las implicaciones de un modelo social en el abordaje de la discapacidad, lo cual se puede resumir en lo siguiente:

- Este modelo enfatiza en la rehabilitación, pero de la sociedad que hará frente a las necesidades de las personas y gestionará las diferencias favoreciendo la diversidad.

- La perspectiva de responsabilidad social de la discapacidad bajo el modelo se situó en una cuestión de derechos humanos, en tanto que es la sociedad la responsable de proveer las adaptaciones para que las personas con discapacidad puedan ejercer su rol y desempeñarse en igualdad de oportunidades.

- La solución exige la acción social, y la sociedad tiene la responsabilidad colectiva de realizar las modificaciones necesarias en el entorno para facilitar la plena participación en todas las esferas de la vida social de las personas con discapacidad (7).

\section{EL MODELO POLÍTICO ACTIVISTA, UNA CUESTIÓN DE DERECHOS DE LAS PERSONAS CON DISCAPACIDAD}

El modelo político activista se fundamenta en la necesidad de la reivindicación de derechos humanos de las personas con discapacidad, que les permita definir su identidad bajo el derecho a formar un colectivo integrado por individuos de iguales características, y derecho a crear unas condiciones de vida positivas sobre la base de la propia identidad y aceptación.

Este modelo emerge de la estructura de la teoría sociocrítica, que se define como una extensión del modelo social en el cual se incorporan las luchas por los derechos de las personas con discapacidad, partiendo del supuesto de que las personas con discapacidad son un grupo minoritario que ha sido histórica y sistemáticamente explotado por la sociedad con relación a la 
educación, transporte y la vivienda, entre otros. Desde esta perspectiva, estas características son consecuencias de las actitudes de la población, de las inexistentes políticas sociales relacionadas con la sociedad. En este sentido la tendencia político-activista ubica el problema no en la falta de movilidad generada por la discapacidad, sino por la incapacidad de la sociedad para dar respuesta a sus necesidades; por esta razón estos modelos centran sus actuaciones no en el campo de la salud sino en los campos político y social (9).

Bajo esta perspectiva, "hablar de discapacidad desde el enfoque de derechos humanos implica un refuerzo jurídico que sobrepasa al modelo social; la idea de una perspectiva de derechos relacionado con el tratamiento de la discapacidad en muchas ocasiones ha sido solamente nombrada sin llegar a concretarse, de manera que todavía en nuestros tiempos las personas con discapacidad tienen dificultades para disfrutar de un trato igualitario con relación a los derechos de las personas" (14).

Al respecto, la Convención Internacional de Derechos de las Personas con Discapacidad (CDPD) describe en su Artículo 1, que su objeto es "promover, proteger y asegurar el goce pleno y en condiciones de igualdad de todos los derechos humanos y libertades fundamentales por todas las personas con discapacidad y promover el respeto de su dignidad inherente". Indica además que para lograr la inclusión desde el enfoque de los derechos humanos es necesario tener en cuenta las siguientes consideraciones:

- Visibilizar la discapacidad desde el enfoque de diversidad y de derechos ya que este último proclama la equiparación de oportunidades, la igualdad y valor de la persona con discapacidad, aspecto fundamental que favorece la eliminación de estereotipos e imaginarios equivocados y negativos.

- Incluir los conceptos de discriminación, sus causas y los mecanis- 
mos que garantizan la igualdad de todos es fundamental, a fin de erradicar las desigualdades estructurales fundamentadas en barreras físicas y mentales, logrando un marco común, el cual sea entendido y compartido por todos los interesados e involucrados en el tema de discapacidad.

- Reconocer la discapacidad desde este modelo, crea un marco dinámico y novedoso que favorece la superación del modelo médico o rehabilitador.

Bajo esta perspectiva, este modelo se encuentra íntimamente relacionado con la asunción de ciertos valores intrínsecos a los derechos humanos y aspira a potenciar el respeto por la dignidad humana, la igualdad y la libertad personal, propiciando la inclusión social, y sentándose sobre la base de determinados principios: autonomía personal, no discriminación, accesibilidad universal, normalización del entorno, diálogo civil, entre otros. Parte de la premisa de que la discapacidad es una construcción y un modo de opresión social y resultado de una sociedad que no considera ni tiene presente a las personas con discapacidad (13).

\section{EL MODELO RELACIONAL, UNA MIRADA BIOPSICOSOCIAL DE LA DISCAPACIDAD}

A partir de este modelo o enfoque relacional, la discapacidad da un paso importante de integración conceptual porque intenta trascender los límites de los dos modelos polarizados: el individual y el social, y provee una perspectiva distinta de los estados de la salud bajo factores sociales y biológicos que incluye dentro de un enfoque "biopsicosocial" (15).

Desde esta perspectiva se analizan las múltiples relaciones que se establecen en la vida de las personas con discapacidad, las cuales obedecen a factores tanto inherentes al propio sujeto como externos creados por la 
sociedad. Lo anterior en respuesta a que la experiencia de la discapacidad es única para cada individuo, no solo porque la manifestación concreta de la enfermedad, desorden o lesión es única, sino porque la condición de salud estará influida por una compleja combinación de factores (desde las diferencias personales, experiencias, antecedentes y bases emocionales, construcciones simbológicas e intelectuales, hasta el contexto físico, social y cultural en el que la persona vive), ello también porque no solo las experiencias individuales de discapacidad son únicas, sino porque las percepciones y actitudes hacia la discapacidad son relativas, ya que están sujetas a interpretaciones culturales que dependen de valores, contexto, lugar y tiempo sociohistórico, así como de la perspectiva del estatus social del observador (4).

En este sentido, bajo el modelo relacional, se sitúa el modelo biopsicosocial bajo el cual la discapacidad se permea como un proceso continuo de ajuste entre las capacidades del individuo con una condición de salud específica, y los factores externos que representan las circunstancias en las que viven las personas, incluyendo las expectativas y exigencias de su entorno. En este contexto, la discapacidad deja de tener un carácter individual y pasa a ser una problemática que atañe a diversos sectores de la sociedad, ya que afecta directamente a la familia y a la comunidad (15).

El modelo biopsicosocial busca articular los modelos biológico y social de la discapacidad, situando la salud y sus componentes en términos de niveles de funcionamiento, superando la mirada única del impacto resultante de enfermedades en el nivel corporal del individuo al concebirla como una situación que involucra los entornos familiar, social, político, económico y cultural (9).

El modelo biopsicosocial de la discapacidad es un rango de aplicación universal de los seres humanos y no un identificador único de un grupo social, 
es decir, el principio del universalismo implica que los seres humanos tienen, de hecho, o en potencia, alguna limitación en su funcionamiento corporal, personal o social asociado a una condición de salud. De hecho, la discapacidad, y sus dimensiones, son siempre relativas a las expectativas sobre el funcionamiento de las personas (qué se espera o no que haga una persona) es una clara consecuencia del universalismo (4).

Bajo este modelo, el funcionamiento y la discapacidad son dos caras de la misma moneda que dependen de las condiciones de salud y la influencia de los factores contextuales. Un lado está representado por el funcionamiento humano el cual se organiza en tres niveles, el primero representado por las funciones y estructuras corporales (partes anatómicas y sus componentes, funciones fisiológicas de los sistemas corporales); el segundo por la capacidad del sujeto para desarrollar actividades que le permiten enfrentar las diversas tareas de la vida cotidiana, y el tercero determinado por la posibilidad de participación social del ser humano en diferentes contextos y que determinan la implicación en situacienes vitales o el desempeño en escenarios reales. El otro lado de la moneda lo representa la discapacidad, la cual surge cuando se altera el funcionamiento humano, en cualquiera de sus tres niveles, el corporal, el personal y el social, determinado por la influencia de los factores contextuales.

Los factores contextuales representan los antecedentes completos de la vida del individuo, que ejercen un impacto sobre él, en el cuerpo, en la persona o en su relación con la sociedad; estos factores se definen con relación a la vida personal y al ambiente en el que se desarrolla la persona; el análisis de estos factores, sitúa aspectos relacionados con la vida de un sujeto y de su estilo y modo de vida, así como aspectos constitutivos de su personalidad y de su estado afectivo y emocional.

Los factores de índole ambiental se analizan en dos niveles, el nivel indi- 
vidual y el nivel social. El nivel individual está representado por el contexto/entorno inmediato del individuo, incluyendo espacios tales como el hogar, el lugar de trabajo o la escuela. El nivel social hace referencia a las estructuras sociales formales e informales, servicios o sistemas globales existentes en la comunidad o cultura, que tiene un efecto en los individuos.

A pesar de que el modelo biopsicosocial realiza un análisis relacional de los factores contextuales con los niveles de funcionamiento, no profundiza sobre los tipos de factores externos, sus relaciones internas y roles para llegar a definir la discapacidad; por este motivo Wang, Badley y Gignac decidieron categorizar estos tipos de relaciones de los factores contextuales con los modelos conceptuales de la siguiente manera (1):

- Factores contextuales moderadores: son aquellos que limitan la realización de una actividad y por ende la participación; un ejemplo de ello es el ambiente físico construido, la educación, los servicios de salud, entre otros. Según sea el nivel de interacción del factor moderador con la limitación para realizar la actividad o participar, se observarán tres tipos de factores moderadores: Factor contextual moderador mutuo (el que influye directamente sobre la participación, Por ejemplo, un persona con una limitación funcional pero con un nivel determinado de educación, factor que puede incidir positivamente en su mayor participación en el trabajo); Factor contextual moderador contingente (el que posibilita la participación aun cuando no exista limitación para realizar la actividad); Factor contextual moderador de respuesta (tiene incidencia directa con la limitación en la actividad. Por ejemplo, audífono mejora la acción de escuchar).

- Factores contextuales mediadores: son variables relacionadas con aspectos psicológicos o emocionales que inciden en la persona de 
manera positiva o negativa contribuyendo o limitando la actividad; al presentarse se convierte en una interferencia para la participación. Por ejemplo:

- Factores contextuales distractores: se encuentran relacionados con la participación, pero también dependen en menor proporción de la limitación para realizar la actividad. Por ejemplo se supera una limitación en una actividad para realizar un trabajo, pero factores de discriminación racial o de género impiden la participación. Es el caso de una persona con discapacidad motora cuyas funciones requieran su trabajo permanente frente a un computador; sin embargo, el hecho de ser mujer la descalifica para el empleo.

- Factores contextuales independientes: estos no se relacionan con discapacidad, limitaciones funcionales o participación; pueden ser externos y aun así afectar los procesos de participación. Por ejemplo, la tasa de desempleo, los indicadores de educación, los perfiles epidemiológicos, los niveles de pobreza, los cuales tienen directa incidencia para que una persona llegue a una condición de discapacidad; se convierten en un importante factor, en países en desarrollo, para los procesos de determinar la discapacidad dada su relevante influencia en la participación (16).

- Bajo este modelo, la discapacidad se permea como un proceso continuo de ajuste entre las capacidades del individuo con una condición de salud específica, y los factores externos que representan las circunstancias en las que viven las personas, incluyendo las expectativas y exigencias de su entorno. En este contexto, la discapacidad deja de tener un carácter individual y pasa a ser una problemática que atañe a diversos sectores de la sociedad, ya que afecta directamente la familia y la comunidad (15). 


\section{LA DISCAPACIDAD DESDE EL ENFOQUE DE CAPACIDADES Y FUNCIONAMIENTO HUMANO}

En el marco de la relación entre capacidad y funcionamiento con calidad de vida y bienestar de la persona con discapacidad se ha promovido un enfoque definido por Amartya Sen, que busca darle sentido a la diversidad de características propias y externas de las personas, como determinantes de lo que las personas pueden realmente hacer o ser, como parte de su desarrollo humano.

La noción más primitiva de este enfoque se refiere a los "funcionamientos". Estos representan partes del estado de una persona: en particular, las cosas que logra hacer o ser al vivir. El enfoque se basa en una visión de la vida como combinación de varios "quehaceres y seres", en el que la calidad de vida debe evaluarse en términos de la capacidad para lograr funcionamientos valiosos. Algunos funcionamientos son muy básicos, como estar nutrido adecuadamente, tener buena salud, etc., y a todos estos se les pueden asignar ponderaciones altas, por razones obvias. Otros pueden ser más complejos, pero seguir siendo ampliamente apreciados, como alcanzar la autodignidad o integrarse socialmente (3).

Toboso citando a Sen (8), expresa que el bienestar es ahora una valoración de las condiciones de vida constituidas por los funcionamientos. Estos son hechos de la vida personal, efectivos y no meramente hipotéticos, y por tanto aspectos constitutivos de la manera en que la persona vive. Obviamente no ocurre un solo funcionamiento cada vez, sino que la persona funciona simultáneamente de muchas maneras: está alimentado, y sano, y protegido, y lee, y viaja, y participa en la sociedad, etc. Su vida puede ser contemplada así, mediante este conjunto de funcionamientos, el cual define el "estado general" de la persona, su forma de vivir. No es que los funcionamientos sean meros expedientes útiles para hacer una descripción, 
sino que son integrantes mismos de esa forma de vida: "La vida -dice Sen- puede considerarse como un conjunto de funcionamientos interrelacionados, consistentes en estados y acciones". Si los funcionamientos alcanzados constituyen el bienestar de una persona, entonces la capacidad para alcanzar funcionamientos (es decir, todas las combinaciones alternativas que una persona puede elegir), constituirá la libertad de esa persona, sus oportunidades reales para obtener bienestar (3).

Bajo la perspectiva de capacidad, se hace referencia igualmente a los planteamientos de Nussbaum, quien define la capacidad no solo como lo que la persona puede hacer sino también lo que logra ser, bajo la combinación de la potencialidad y las condiciones necesarias para su desempeño.

De este modo, una persona tiene una «capacidad» no solo cuando tiene una potencialidad para realizar una acción, sino cuando puede hacer un uso real de ella. En suma, el concepto de «capacidad» propuesto por Nussbaum incluye una posibilidad o potencialidad, más un entorno material y social apropiado que permita su realización, lo cual implica que las personas tengan garantizados los medios necesarios para hacer uso de las «capacidades». (17)

En esta relación, se presupone como lo plantea García (18), que la discapacidad no es solo el resultado de una limitación física o mental, sino que puede ser generada por la carencia de unas condiciones y un entorno inapropiado para el desarrollo de las potencialidades. Lo anterior significa, siguiendo al autor, que una persona puede estar limitada en un ámbito físico o mental, pero esto no implica que sus «capacidades» estén imposibilitadas.

Esta mirada de la discapacidad desde la relación entre capacidad y entorno, reconfirma la apuesta de la OMS cuando plantea que la discapacidad se permea como una tensión y ajuste permanente entre las capacidades del individuo con una condición de salud específica, y los factores externos que 
representan las circunstancias en las que viven las personas y que median el uso de dichas capacidades en contextos reales.

\section{REFERENCIAS BIBLIOGRÁFICAS}

1. Gómez AC, Cuervo EC. Conceptualización de discapacidad: reflexiones para Colombia. Ediciones Universidad Nacional de Colombia 2007, p.35 ISBN 978-958-701-852-3.

2. Cortés Reyes E, Riveros L T, Pineda-Ortiz G A. Clasificación internacional del funcionamiento, la discapacidad y certificación de discapacidad en Colombia. Rev. Salud Pública. 2013; 15(1):129-137.

3. Vargas DM. Miradas epistemológicas desde distintas perspectivas teóricas sobre la discapacidad. Revista Electrónica Educare. 2012; 16(3): 145-155.

4. Vanegas GJ, Gil OL. La teoría de sistemas y el modelo bio-psico-social. Hacia la Promoción de la Salud. 2007; 12:51-61.

5. Peñas FO. Referentes conceptuales para la comprensión de la discapacidad. Rev. Fac. Med. 2013; 61(2):205-212.

6. Moreno FM. Importancia de los modelos conceptuales y teorías de enfermería: experiencia de la Facultad de Enfermería de la Universidad de La Sabana. Aquichan. 2005; 5(1).

7. Velázquez DE. Reflexiones epistemológicas para una sociología de la discapacidad. Revista Sociológica de Pensamiento Crítico. 2009; 3(2):85-99.

8. Toboso MM, Arnau RM. La discapacidad dentro del enfoque de capacidades y funcionamientos de Amartya Sen. Araucaria. Revista Iberoamericana de Filosofía, Política y Humanidades. 2008; 20:64-94.

9. Universidad Autónoma de Manizales. Proyecto Educativo Programa de Maestría en Discapacidad. 2013.

10. UIPC-IMSERSO. Unidad 2. Evolución histórica de los modelos en los que se fundamenta la discapacidad. 
11. Conde MR. Evolución del concepto de discapacidad en la sociedad contemporánea: de cuerpos enfermos a sociedades excluyentes. Praxis Sociológica. 2014; 18:155-175.

12. Organización Mundial de la Salud. Informe mundial sobre la discapacidad. 2011.

13. Victoria MJ. El modelo social de la discapacidad: una cuestión de derechos humanos. Bol.Mex.Der.Comp. 2013; 46(138):1093-1109.

14. Cuenca PG. Derechos humanos y modelos de tratamiento de la discapacidad. Instituto de Derechos Humanos-Bartolomé de las Casas. 2011; 3:16. ISSN: 1989-8797.

15. Lidón LH. Discapacidad y Observaciones Generales de los comités de derechos humanos de la ONU: una relación asimétrica entre la invisibilidad, el modelo médico y el modelo de derechos humanos. Revista Española de Discapacidad. 2013; 1(1): 47-72.

16. Organización Mundial de la Salud. Clasificación Internacional del Funcionamiento, de la Discapacidad y de la Salud-CIF. 2001.

17. Herazo BY, Domínguez AR. Correlación entre pobreza extrema y discapacidad en los departamentos de Colombia. Cienc. Innov. Salud. 2013; 1(1):11-17.

18. García G N. El «enfoque de las capacidades» de Nussbaum y el concepto de «discapacidad». Revista Légein. 2009; 9:101-119 ISSN 1794-5291.

\footnotetext{
Cómo citar este capítulo:

Pinillos Patiño Y, Naranjo Aristizábal MM. (2018). Modelos conceptuales que explican la discapacidad: de la teoría a la comprensión del funcionamiento. In Pinillos Patiño Y, Herazo Beltrán Y, Vidarte Claros JA, Crissién Quiroz EM, Suárez Palacio D, García Puello F, et al. Caracterización de la discapacidad en el distrito de Barranquilla. Una mirada conceptual y experiencial. Barranquilla: Universidad Simón Bolívar, p. 53-74.
} 